EESTI NSV TEADUSTE AKADEEMIA TOIMETISED. 28. KOIDE FOOSIKA * MATEMAATIKA. 1979, NR. 4

ИЗВЕСТИЯ АКАДЕМИИ НАУК ЭСТОНСКОИ ССР. ТОМ 28 ФИЗИКА * МАТЕМАТИКА. 1979, № 4

\title{
HODOGRAPH TRANSFORMATIONS AND HODOGRAPH INVARIANT DIFFERENTIAL EQUATIONS
}

\author{
(Presented by H. Keres)
}

\begin{abstract}
A phenomenon present in many physical problems - hodograph invariance of partial differential equations - is considered for two-dimensional submanifolds in three- and four-dimensional spaces. This means that the equation keeps its shape when interchanging the roles of any of the functions and arguments, i.e. under a hodograph transformation. For that purpose unified formulations for hodograph transformations are obtained. A method for the formation of hodograph invariant equations is shown. The well-known scalar Born-Infeld equation and its two-component generalization prove to be based on hodograph quasi-invariant expressions.
\end{abstract}

\section{Introduction}

In an earlier paper $\left[{ }^{1}\right]$ the scalar Born-Infeld equation in an arbitrary space dimension was reached departing from quite a general physical problem. The movement of a generalized relativistic string was presented in a fixed Cartesian co-ordinate system. The interior variables of the string could be eliminated, i. e. the object proved to be a so-called sympleon. At that some Cartesian co-ordinates had to be considered as functions and the rest as arguments. The functions might be chosen quite arbitrarily and the equation obtained should not materially depend on this choice.

Such possible property of partial derivative differential equations hodograph invariance - is the investigation object of this paper. it may be expected that in essence many physical problems must be hodograph invariant. For instance, the shape of the equation describing minimality or constant total curvature of a membrane cannot depend on the orientation of co-ordinates. The same is valid for any movement of a free particle.

Our basic definitions are the following.

Hodograph transformation is an interchange of any of the functions and arguments.

Hodograph invariant expression is an expression of partial derivatives which retains its shape after a hodograph transformation. If the conserved shape arises as a factor in the transformed form, the expression is called hodograph quasi-invariant.

Hodograph invariant equation appears if either a hodograph invariant or a hodograph quasi-invariant expression is equated to zero. 
Hodograph transformations have been used for a long time as auxiliary means for solving differential equations. Here the attempt is made to show that they may have a physical significance as well. In this paper the problem of constructing first- and second-order hodograph invariant partial derivative differential equations is dealt with. For this purpose a more general approach to hodograph transformations is needed. At that we are confined to second derivatives and two cases of dimensions: a two-dimensional surface in three-dimensional space and a two-dimensional surface in four-dimensional space in the Euclidean metric with reference to other metrics.

\section{Two-surface in three-space}

Let there be a differentiable two-dimensional surface $R^{2}$ in three-dimensional space $R^{3}$ with the Cartesian co-ordinates ${ }_{i} X(i=1,2,3)$. The surface can be presented by any of the three different explicit forms

$$
{ }_{i} X={ }_{i} X\left({ }_{j} X,{ }_{k} X\right) \text {. }
$$

This description will further be called $i$-representation after the choice of the function. Here all three representations will be considered parallelly without giving preference to any one in particular.

Hodograph transformations are the transitions between representations. For a function itself a hodograph transformation means taking the respective inverse function. For partial derivatives the hodograph transformations between $i$ - and $j$-representations are known as $\left.{ }^{2}\right]$ (no summation! $i \neq j \neq k \neq i$ )

$$
\begin{gathered}
{ }_{i} X_{j}={ }_{j} X_{i}^{-1}, \quad{ }_{i} X_{k}=-{ }_{j} X_{k} / X_{j} X_{i} ; \\
{ }_{i} X_{j j}=-{ }_{j} X_{i}^{-3}{ }_{j} X_{i i}, \quad{ }_{i} X_{j k}={ }_{j} X_{i}^{-3}\left({ }_{j} X_{k j}{ }_{j} X_{i i}-{ }_{j} X_{i}{ }_{j} X_{i k}\right), \\
{ }_{i} X_{k k}={ }_{j} X_{i}^{-3}\left(2 X_{j} X_{i}{ }_{j} X_{k} X_{j} X_{i k}-{ }_{j} X_{i}{ }_{i}{ }_{j} X_{k k}-{ }_{j} X_{k}{ }_{j}{ }_{j} X_{i i}\right),
\end{gathered}
$$

where

$$
{ }_{a} X_{b} \equiv \partial_{a} X / \partial_{b} X ; \quad{ }_{a} X_{b c} \equiv \partial_{a}^{2} X / \partial_{b} X \partial_{c} X .
$$

These formulae can be unified, defining the quantities

$$
\begin{aligned}
{ }_{i} R_{j} & =\left\{\begin{array}{cl}
1, & \text { if } i=j ; \\
-{ }_{i} X_{j}, & \text { if } i \neq j,
\end{array}\right. \\
{ }_{i} S_{j k} & =\left\{\begin{array}{cc}
0, & \text { if } i=j \text { or } i=k, \\
-{ }_{i} X_{j k}, & \text { if } j \neq i \neq k,
\end{array}\right.
\end{aligned}
$$

which possess the following symmetry properties:

$$
{ }_{i} R_{j}={ }_{j} R_{i}^{-1}, \quad{ }_{i} S_{j k}={ }_{i} S_{k j} .
$$

Now a hodograph transformation can be written without any limitation of the values of the indices:

$$
\begin{aligned}
& { }_{i} R_{k}={ }_{j} R_{k} /{ }_{j} R_{i}, \\
& { }_{i} S_{k l}={ }_{j} R_{i}^{-3}{ }_{j} R_{[i j} R_{[i j} S_{k] l]},
\end{aligned}
$$

where in the latter there are independent alternations between the two pairs of indices: 


$$
A_{[a b]} \equiv A_{a b}-A_{b a} .
$$

Note that the condition that a differential line element $\left(d_{1} X, d_{2} X, d_{3} X\right)$ lies on the surface

$$
d_{i} X={ }_{i} X_{j} d_{j} X+{ }_{i} X_{k} d_{k} X
$$

can now be written as

$$
\sum_{l}{ }_{i} R_{l} d_{l} X=0
$$

When considering hodograph invariance, one can easily verify that various hodograph invariant expressions are obtainable by forming from $R$ and $S$ the expressions symmetric with respect to all three values of the indices separately for the left- and right-side ones followed by the transformation into a certain representation.

However, in physics a covariance with respect to $\mathrm{O}(2)(\mathrm{O}(1.1)$ in a pseudo-Euclidean case) rotations is usually needed in addition. It is easy to see that a simultaneous covariance in all representations can be achieved by observing the vector indices on the right; for scalar expressions all the right indices must be summed up in pairs.

To build up hodograph invariant equations quasi-invariant expressions will suffice. This is why one need not always sum up the left (representation) indices, since quasi-invariance can be guaranteed by other, more complicated principles. Equation (1.7) suggests the first-order hodograph quasi-invariant

$$
{ }_{i} I(1,2,2)=\sum_{k} R_{k}^{2},
$$

where the numbers in brackets label in sequence: 1 - order, $2-$ degree, 2 - the so-called class, i. e. the power of ${ }_{j} R_{i}^{-1}$ that arises at the hodograph transformation $i \rightarrow j$ :

$$
{ }_{i} I(1,2,2)={ }_{j} R_{i}^{-2}{ }_{j} I(1,2,2) .
$$

Denoting the function by $z$ and the arguments by $x, y$, this quasiinvariant takes the form*

$$
{ }_{2} I(1,2,2)=1+z_{x}^{2}+z_{y}^{2} .
$$

The second-order quasi-invariants are

$$
\begin{aligned}
& { }_{i} I(2,1,3)=\sum_{j, k} R_{i j i} R_{[k}{ }^{i} S_{j] k}, \\
& { }_{i} I(2,2,4)=\sum_{j, k} S_{j[j}{ }^{i} S_{k] k} .
\end{aligned}
$$

Their explicit forms are

$$
\begin{aligned}
& { }_{z} I(2,1,3)=\left(1+z_{x}^{2}\right) z_{y y}-2 z_{x} z_{y} z_{x y}+\left(1+z_{y}^{2}\right) z_{x x}, \\
& { }_{z} I(2,2,4)=z_{x x} z_{y y}-z_{x y}^{2} .
\end{aligned}
$$

* Note that $\left(1+z_{x}^{2}+z_{y}^{2}\right)^{1 / 2} d x d y$ equals the area of an infinitesimal element of the surface. 
The hodograph invariant equation

$$
{ }_{i} I(2,1,3)=0
$$

is the known Lagrange equation which describes minimal surfaces $\left[{ }^{3}\right]$. This is a Euclidean analogue of the scalar Born-Infeld equation.

In principle one can build an infinity of equations out of these expressions. It should only be kept in mind that the resulting expression must have a well-defined class. For instance, the equation of class 4

$$
I(2,2,4)-2 k I^{2}(1,2,2)=0
$$

can be formed, which describes surfaces with the constant total curvature $k$ [ $\left.{ }^{3}\right]$.

If the metric is not Euclidean, the covariant summations over the vector indices in equations $(1.11),(1.12),(1.15),(1.16)$ must be performed through the corresponding metric tensor:

$$
\sum_{a} \mathrm{~A}_{a a} \rightarrow \sum_{a, b} g_{a b} A_{a b} .
$$

Thus, for the pseudo-Euclidean metric, where

$$
g=\left(\begin{array}{rrr}
1 & 0 & 0 \\
0 & -1 & 0 \\
0 & 0 & -1
\end{array}\right)
$$

quasi-invariants $(1.14),(1.17),(1.18)$ take the forms

$$
\begin{aligned}
& { }_{y} I(1,2,2)=y_{t}^{2}-y_{x}^{2}-1, \\
& { }_{y} I(2,1,3)=\left(1-y_{t}^{2}\right) y_{x x}+2 y_{t} y_{x} y_{t x}-\left(1+y_{x}^{2}\right) y_{t t}, \\
& { }_{y} I(2,2,4)=y_{x t}^{2}-y_{t t} y_{x x},
\end{aligned}
$$

but in the $t$-representation

$$
\begin{aligned}
& { }_{t} I(1,2,2)=1-t_{x}^{2}-t_{y}^{2}, \\
& { }_{t} I(2,1,3)=\left(t^{2}-1\right) t_{x x}-2 t_{x} t_{y} t_{x y}+\left(t_{x}^{2}-1\right) t_{y y}, \\
& { }_{t} I(2,2,4)=t_{x x} t_{y y}-t_{x y}^{2},
\end{aligned}
$$

where the scalar Born-Infeld expression can be recognized.

\section{Two-surface in four-space}

This case is applicable in particle physics for the description of a relativistic string in space-time. If the motion is free, the equation must be of the same form in all representations, i. e. hodograph invariant.

A two-dimensional surface in a four-dimensional space is presented by two relations between four co-ordinates. An explicit form contains two functions and two independent arguments. Thus, a representation must be labelled by two indices, corresponding to the choice of the functions.

Let us first consider again the case of the Euclidean metric with co-ordinates $\alpha X(\alpha=1,2,3,4)$. The partial derivatives can be represented by the quantities $(\alpha \neq \beta)$ 


$$
\begin{aligned}
{ }_{\alpha \beta} T_{\mu} \equiv \begin{cases}1, & \text { if } \mu=\alpha, \\
0, & \text { if } \mu=\beta, \\
-\partial_{\alpha} X / \partial_{\mu} X, & \text { if } \alpha \neq \mu \neq \beta,\end{cases} \\
{ }_{{ }_{\beta} \beta} V_{\mu v} \equiv \begin{cases}0, & \text { if any of } \mu, v=\alpha, \beta, \\
-\partial^{2}{ }_{\alpha} X / \partial_{\mu} X \partial_{\nu} X, & \text { if } \mu \neq \alpha, \beta ; \nu \neq \alpha, \beta .\end{cases}
\end{aligned}
$$

Note that every partial derivative depends on the choice of other functions. This is why, for instance,

$$
{ }_{12} T_{4}=\partial_{1} X / \partial_{4} X=d_{1} X /\left.d_{4} X\right|_{d_{3} X=0}
$$

and

$$
{ }_{13} T_{4}=\partial_{1} X / \partial_{4} X=d_{1} X /\left.d_{4} X\right|_{d_{2} X=0}
$$

differ from each other.

All hodograph transformations can be calculated by expressing all differentials in terms of two independent arbitrary differentials $d u$, $d v$ on the surface:

$$
d_{\mu} X={ }_{\mu} X_{u} d u+{ }_{\mu} X_{v} d v .
$$

In that case the differential of the remaining argument must be taken zero:

$$
d_{v} X={ }_{v} X_{u} d u+{ }_{v} X_{v} d v=0
$$

which can be used for the elimination of $d v$. The remaining differential $d u$ cancels out from derivatives. For instance, in the 1,2-representation

$$
\partial_{1} X / \partial_{3} X={ }_{4} X_{[u 1} X_{v]} /{ }_{4} X_{[u 3} X_{v]} .
$$

By comparing these expressions with each other, the following hodograph transformation rules can be found.

When exchanging the first indices $\alpha \rightarrow \gamma$ :

$$
\begin{gathered}
{ }_{\alpha \beta} T_{\mu}={ }_{\nu \beta} T_{\alpha}^{-1}{ }_{\alpha \beta} T_{\mu}, \\
{ }_{\alpha \beta} V_{\mu \nu}={ }_{\nu \beta} T_{\alpha \nu \beta}^{-3} T_{[\alpha \gamma \beta} T_{[\alpha \nu \beta} V_{\mu] \nu]},
\end{gathered}
$$

which, as expected, is of the same form as in the previous three-dimensional case because the remaining function ${ }_{\beta} X$ is not involved.

When the remaining function is exchanged, $\beta \rightarrow \delta$, then

$$
\begin{gathered}
\alpha_{\beta} T_{\mu}={ }_{\delta \alpha} T_{\beta \delta \alpha}^{-1} T_{[\beta \alpha \delta} T_{\mu]}, \\
{ }_{\alpha \beta} V_{\mu \nu}={ }_{\delta \alpha} T_{\beta \delta \alpha}^{-3} T_{[\beta \delta \alpha} T_{[\beta}\left({ }_{\delta \alpha} T_{\beta \alpha \delta} V_{\mu] v]}-{ }_{\alpha \delta} T_{\beta \delta \alpha} V_{\mu] v]}\right) .
\end{gathered}
$$

And when exchanging both $\alpha \beta \rightarrow \gamma \delta$,

$$
\begin{aligned}
{ }_{\alpha \beta} T_{\mu} & =\left({ }_{\gamma \delta} T_{[\alpha \delta \gamma} T_{\beta]}\right)^{-1}{ }_{\gamma \delta} T_{[\mu \delta \gamma} T_{\beta]}, \\
{ }_{\alpha \beta} V_{\mu \nu} & =\left({ }_{\gamma \delta} T_{[\alpha \delta \gamma} T_{\beta]}\right)^{-3} \sum_{\rho, \sigma, \tau, \nu, \xi, \eta} \delta_{\alpha \beta \mu}^{\rho \sigma \tau} \delta_{\alpha \beta \nu \gamma \delta}^{\delta \xi \eta} T_{\rho \gamma \delta} T_{\delta \delta \gamma} T_{\sigma \delta \gamma} T_{\xi} \times \\
& \times\left({ }_{\gamma \delta} T_{\beta \delta \gamma} V_{\tau \eta}-{ }_{\delta \gamma} T_{\beta \gamma \delta} V_{\tau \eta}\right),
\end{aligned}
$$

where $\delta$ is the alternator which equals 1 if its lower indices equal an even permutation of the upper ones, -1 , if the permutation is odd or 
0 in other cases and when there are equal indices among either upper or lower sets. By the way,

$$
A_{[a b]} \equiv \sum_{c, d} \delta_{a b}^{c d} A_{c d}
$$

Hodograph invariants can be found separately for the three kinds of hodograph transformations. As equations (2.8) and (2.9) are identical with those in the three-dimensional case, the corresponding invariants and quasi-invariants are identical too. Thus,

$$
\sum_{\mu \beta} T_{\mu}^{2}
$$

is a quasi-invariant under an exchange of the first index $\alpha \rightarrow \gamma$, but not under the other kinds of exchanges. The second-order quasi-invariants are also identical to (1.15) and (1.16) if a respective exchange is performed.

As far as the invariant expressions are concerned, they can always be built for each kind of transformations by any symmetrization of the respective index (or indices) over all four values.

As to the exchange $(2.10),(2.11)$ only, we could not find any specific quasi-invariant.

For the full interchange of the arguments and functions (2.12), (2.13) there exists the quasi-invariant

$$
{ }_{\alpha \beta} I(1,2,2)=\sum_{\mu}\left({ }_{\alpha \beta} T_{\mu}^{2}+{ }_{\beta \alpha} T_{\mu}^{2}\right)-2,
$$

where class 2 indicates the power of $\left({ }_{\alpha \beta} T_{[\rho} \beta \alpha T_{\sigma]}\right)^{-1}$ at the transformation $\alpha \beta \rightarrow \varrho \sigma$. Its explicit form is

$$
{ }_{\varphi \psi} I(1,2,2)=\varphi_{x}^{2}+\varphi_{y}^{2}+\psi_{x}^{2}+\psi_{y}^{2} .
$$

We can also show a second-order system for this case. If to define the quantities

$$
\begin{aligned}
& Q_{\alpha}={ }_{\alpha \beta} T_{\rho \alpha \beta} V_{\sigma \sigma}-\left({ }_{\alpha \beta} T_{\sigma}+{ }_{\beta \alpha} T_{\rho}\right)_{\alpha \beta} V_{\rho \sigma}+{ }_{\beta \alpha} T_{\sigma \alpha \beta} V_{\rho \rho}, \\
& Q_{\beta}={ }_{\alpha \beta} T_{\rho \beta \alpha} V_{\sigma \sigma}-\left({ }_{\alpha \beta} T_{\sigma}+{ }_{\beta \alpha} T_{\rho}\right)_{\beta \alpha} V_{\rho \sigma}+{ }_{\beta \alpha} T_{\sigma \beta \alpha} V_{\rho \rho},
\end{aligned}
$$

then it is

$$
\begin{aligned}
& { }_{\alpha \beta} I^{\prime}(2,1,3)={ }_{\alpha \beta} T_{\rho} Q_{\alpha}-{ }_{\beta \alpha} T_{\rho} Q_{\beta} \pm i Q_{\alpha}, \\
& { }_{\beta \alpha} I^{\prime}(2,1,3)={ }_{\alpha \beta} T_{\sigma} Q_{\alpha}-{ }_{\beta \alpha} T_{\sigma} Q_{\beta} \pm i Q_{\beta},
\end{aligned}
$$

which may be written explicitly as

$$
\begin{aligned}
{ }_{\varphi} I^{\prime}(2,1,3) & =-\varphi_{x} \psi_{x} \psi_{y y}+\varphi_{x}\left(\psi_{y}+\varphi_{x}\right) \psi_{x y}-\varphi_{x} \varphi_{y} \psi_{x x}+ \\
& +\psi_{x}^{2} \varphi_{y y}-\psi_{x}\left(\psi_{y}-\psi_{x}\right) \varphi_{x y}+\psi_{x} \varphi_{y} \varphi_{y y} \pm \\
& \pm i\left[\psi_{x} \psi_{y y}-\left(\psi_{y}+\varphi_{x}\right) \psi_{x y}+\varphi_{y} \psi_{x x}\right] \\
\psi_{\varphi} I^{\prime}(2,1,3) & =-\varphi_{y} \psi_{x} \psi_{y y}+\varphi_{y}\left(\psi_{y}+\varphi_{x}\right) \psi_{x y}-\varphi_{y}^{2} \psi_{x x}+ \\
& +\psi_{y} \psi_{x} \varphi_{y y}-\psi_{y}\left(\psi_{y}-\psi_{x}\right) \varphi_{x y}+\psi_{y} \varphi_{y} \varphi_{x x} \pm \\
& \pm i\left[\psi_{x} \varphi_{y y}-\left(\psi_{y}+\varphi_{x}\right) \varphi_{x y}+\varphi_{x} \varphi_{x x}\right] .
\end{aligned}
$$


Next, the invariance under all three transformations is considered. It can be guessed that this is the most important case for physical problems.

For this purpose transformation equations must be unified. Let us define new representative quantities for the derivatives

$$
\begin{gathered}
{ }_{\alpha \beta}^{\rho} \widetilde{T}_{\mu} \equiv_{\beta \alpha} T_{[\rho \alpha \beta} T_{\mu]}, \\
{ }_{\alpha \beta}^{\rho} \widetilde{V}_{\mu \nu} \equiv_{\beta \alpha} T_{\rho \alpha \beta} V_{\mu v}-{ }_{\alpha \beta} T_{\rho \beta \alpha} V_{\mu v} .
\end{gathered}
$$

They are anti-symmetric with respect to $\alpha, \beta$ (which is natural because the concept of a representation is neutral to the arrangement of these and in principle they cannot be equal) and (2.20) is anti-symmetric with respect to $\mathrm{Q}$ and $\mu$.

Now, using $(2.20)$ and (2.21), the transformation equations (2.8)-(2.13) can be joined together for an arbitrary exchange $\alpha \beta \rightarrow \tau \vartheta$ :

$$
\begin{aligned}
& { }_{\alpha \beta}^{\rho} \widetilde{T}_{\mu}={ }_{\tau{ }^{\beta}}^{\beta} \widetilde{T}_{\alpha \tau \tau^{\rho}}^{-1} \widetilde{T}_{\mu},
\end{aligned}
$$

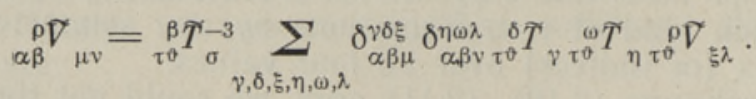

One can see a certain similarity with equations (1.7) and (1.8). Hence it may be expected that the corresponding quasi-invariants can be built as well. The additional index $\mathrm{e}$ seems to allow a building of four parallel independently transforming equations. It would be really so if one endeavours to conserve the shape of the equation in terms of $T$ and $\widetilde{\nabla}$ instead of the derivatives. Fortunately the invariance in terms of derivatives can be achieved as well.

A first-order quasi-invariant is

$$
{ }_{\alpha \beta} T(1,4,2)=\sum_{\mu \rho}{ }_{\alpha \beta}^{\rho} \widetilde{T}_{\mu}^{2},
$$

where quasi-invariance with respect to derivatives is achieved by additional summing up over $\mathrm{e}$. An explicit form for it is

$$
{ }_{\varphi \psi} I(1,4,2)=2\left[1+\varphi_{x}^{2}+\varphi_{y}^{2}+\psi_{x}^{2}+\psi_{y}^{2}+\left(\varphi_{x} \psi_{y}-\varphi_{y} \psi_{x}\right)^{2}\right] .
$$

Another possibility to deal with $\varrho$ is to form a system of equations after its values. This method will be used for other quasi-invariants. Under a hodograph transformation an equation of the system changes into a linear combination of the equations of the corresponding system. Thus, instead of $(2.25)$ there will be the system

$$
\left\{\begin{array}{c}
1+\varphi_{x}^{2}+\varphi_{y}^{2}=0 \\
1+\psi_{x}^{2}+\psi_{y}^{2}=0 \\
\varphi_{x} \psi_{x}+\varphi_{y} \psi_{y}=0
\end{array}\right.
$$

Next, an invariant system, equivalent to (1.19), is

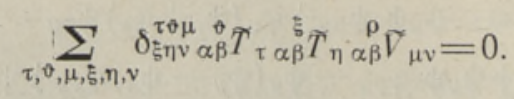

It is reducible to a system of two equations 


$$
\left\{\begin{array}{l}
\left(1+\varphi_{x}^{2}+\psi_{x}^{2}\right) \varphi_{y y}-2\left(\varphi_{x} \varphi_{y}+\psi_{x} \psi_{y}\right) \varphi_{x y}+\left(1+\varphi_{y}^{2}+\psi_{y}^{2}\right) \varphi_{x x}=0 \\
\left(1+\varphi_{x}^{2}+\psi_{x}^{2}\right) \psi_{y y}-2\left(\varphi_{x} \varphi_{y}+\psi_{x} \psi_{y}\right) \psi_{x y}+\left(1+\varphi_{y}^{2}+\psi_{y}^{2}\right) \psi_{x x}=0
\end{array}\right.
$$

i. e. a Euclidean analogue of the two-component Born-Infeld equation **. At last, the second-order second-degree invariant system

$$
\sum_{\mu, v}^{\rho \beta \tilde{V}} \underset{\mu[v \alpha \beta}{\rho \tilde{V}_{\mu] v}}=0
$$

reduces to the form

$$
\left\{\begin{array}{l}
\varphi_{x y}-\varphi_{x x} \varphi_{y y}=0 \\
\psi_{x y}^{2}-\psi_{x x} \psi_{y y}=0
\end{array}\right.
$$

A pseudo-Euclidean metric can easily be introduced by including a corresponding metric tensor in every covariant summation.

\section{Conclusion}

Methods for the construction of hodograph-invariant first- and secondorder expressions in the case of a two-dimensional differentiable submanifold in both three- and four-dimensional spaces are shown. In principle, the methods can be developed to both arbitrary orders and arbitrary dimensions. It can be seen in Sec. 2, what kind of additional complications arise if the dimensions of the space and submanifold differ by more than unity. Within our preconditions some questions remain unanswered: Is there any physical significance for an equation in being built of a quasi-invariant instead of an invariant? How to find all the quasi-invariants of a given order? It may be supposed that for the dimensions considered all the second-order quasi-invariants have been obtained, but this has not been proved yet. Are there any more partial quasi-invariants like (2.18) or not? Presumably further generalizations will bring more clarity of these questions.

** The original Born-Infeld equation is a four-component one [ [ $\left.{ }^{4}\right]$. In a one-component case it reduces to the corresponding scalar equation considered here. A natural generalization of the latter, back to many dimensions does not coincide with the original system in the four-dimensional case.

\section{REFERENCES}

1. A ins a a r, A., K i ir a nen, K., Kõ i v, M., ENSV TA Toimet., Füüs. Matem., 27, № 4, 453-455 (1978).

2. Фихтенгольц Г. М., Курс дифференциального и интегрального исчисления, т. I, М., ГИТТЛ, 1951.

3. E is e n hart, L. P., A treatise on the differential geometry of curves and surfaces, Dover Publications, Inc., New York, 1960.

4. Тонне л а М.-А., Основы электромагнетизма и теории относительности, М., Изд-во иностр. лит., 1962.

Academy of Sciences of the Estonian SSR, Institute of Physics 
A. AINSAAR, M. KOIV

\section{HODOGRAAFTEISENDUSED JA HODOGRAAFINVARIANTSED DIFERENTSIAALVORRANDID}

On käsitletud paljude füüsikaliste probleemide puhul esinevat nähtust - osatuletistega diferentsiaalvõrrandi hodograafinvariantsust - kahemõōtmeliste alammuutkondade korral kolme- ja neljamõōtmelises ruumis. Hodograafinvariantsus tähendab, et võrrand säilitab oma kuju juhul, kui vahetada mistahes funktsiooni ja argumendi osad, s. o. kui teha hodograafteisendus. On esitatud hodograafteisenduste unifitseeritud valemid ja hodograafinvariantsete võrrandite moodustamise meetod. IImneb, et tuntud skalaarne Born-Infeldi võrrand ja tema kahekomponendiline üldistus baseeruvad hodograaf-kvaasiinvariantsetel avaldistel.

\section{A. АИНСААР, М. КЫИВ}

\section{ГОДОГРАФ-ПРЕОБРАЗОВАНИЯ \\ И ГОДОГРАФ-ИНВАРИАНТНЫЕ ДИФФЕРЕНЦИАЛЬНЫЕ УРАВНЕНИЯ}

Рассмотрена часто встречающаяся в физических проблемах ситуация - годографинвариантность уравнений в частных производных в случае двумерных подмногообразий, вложенных в трехмерные и четырехмерные пространства. Годограф-инвариантность означает, что уравнение сохраняет свой вид при любой перестановке функций с аргументами, т. е. при годограф-преобразовании. Получены унифицированные выражения годограф-преобразований. Дан метод для образования годограф-инвариантных уравнений. Установлено, что известное скалярное уравнение Борна-Инфельда и его двухкомпонентное обобщение базируютсл на годограф-квазиинвариантных выражениях. 